Check for updates

Cite this: Mater. Adv., 2020 1, 1653

Received 8th June 2020, Accepted 10th August 2020

DOI: 10.1039/d0ma00390e

rsc.li/materials-advances

\section{The effect of ZnO particle lattice termination on the DC conductivity of LDPE nanocomposites $\dagger$}

\author{
M. E. Karlsson, ${ }^{a}$ A. Calamida, ${ }^{a}$ D. Forchheimer, ${ }^{b}$ H. Hillborg, ${ }^{c}$ V. Ström, (D) ${ }^{d}$ \\ J. M. Gardner, (iD e M. S. Hedenqvist ${ }^{a}$ and R. T. Olsson (iD *a
}

\begin{abstract}
The effects of particle surface termination by zinc or oxygen were evaluated for composites containing micro-sized $\mathrm{ZnO}$ particles with rod shapes (17\% oxygen terminations) or ball shapes $(67 \%$ oxygen terminations), and it was found that the rods gave a conductivity $\left(1.2 \times 10^{-16} \mathrm{~S} \mathrm{~m}^{-1}\right)$ half that given by the ball-shaped particles $\left(2.4 \times 10^{-16} \mathrm{~S} \mathrm{~m}^{-1}\right)$. Both composites containing the micro-sized particles showed a conductivity almost two orders of magnitude lower than that of the LDPE reference material $\left(1.2 \times 10^{-14} \mathrm{~S} \mathrm{~m}^{-1}\right)$. When a $5 \mathrm{~nm}$ thick silica coating was applied to the particles, the silica encapsulation eliminated the difference between the particles and resulted in both cases in an increase in conductivity by an order of magnitude to ca. $2 \times 10^{-15} \mathrm{~S} \mathrm{~m}^{-1}$. The conductivity was still lower than that of the pristine polyethylene polymer. It was concluded that neither the particle morphology nor the inter-particle distance ( $1 \mu \mathrm{m}$ for rods and $8 \mu \mathrm{m}$ for balls) had any effect on the conductivity of the composites for identically terminated particles, while demonstrating that the conductivity of these materials relies uniquely on the particle surface terminations. In contrast, a markedly reduced conductivity was observed for composites containing the same particles but terminated with aliphatic hydrocarbon tails, the conductivity for both rod-shaped and ball-shaped particles $\left(1 \times 10^{-16} \mathrm{~S} \mathrm{~m}^{-1}\right)$ being reduced to even lower values than for the pristine particles without surface modification. The same trend was observed with the $25 \mathrm{~nm} \mathrm{ZnO}$ nanoparticles, showing a record low conductivity of $1 \times 10^{-17} \mathrm{~S} \mathrm{~m}^{-1}$ for $3 \mathrm{wt} \%$ nanoparticles with aliphatic hydrocarbon tails. In practical applications, this would permit higher operation voltages than currently employed HVDC cable systems by controlling the resistivity of the composite insulation for various electric fields and temperatures and making it possible to tailor the dielectric design of cable components.
\end{abstract}

\section{Introduction}

Metal oxide nanoparticles are promising as inorganic fillers in low-density polyethylene (LDPE) composites yielding new insulating materials for high voltage direct current (HVDC) cables. $^{1-3}$ These insulating composites have shown a resistivity $c a$. two orders of magnitude higher than that of the already ultra clean LDPE used as insulation in HVDC cables, which after crosslinking is rated up to $640 \mathrm{kV}{ }^{4}$ Ensuring high resistivity of

\footnotetext{
${ }^{a}$ KTH Royal Institute of Technology, School of Engineering Sciences in Chemistry, Biotechnology and Health, Fibre and Polymer Technology, SE-100 44 Stockholm, Sweden. E-mail: rols@kth.se; Fax: +46 8 208856; Tel: +46 87907637

${ }^{b}$ KTH Royal Institute of Technology, Nanostructure Physics, SE-106 91 Stockholm, Sweden

${ }^{c}$ ABB Power Grids Research, SE-721 78 Västerås, Sweden

${ }^{d}$ KTH Royal Institute of Technology, School of Industrial Engineering and Management, Material Science and Engineering, SE-100 44 Stockholm, Sweden

${ }^{e}$ KTH Royal Institute of Technology, School of Engineering Sciences in Chemistry, Biotechnology and Health, Department of Chemistry, SE-114 28 Stockholm, Sweden

$\dagger$ Electronic supplementary information (ESI) available. See DOI: 10.1039/d0ma00390e
}

the insulation, in addition to high breakdown strength and control of space charge accumulation, is one of the main tasks in HVDC cable development. ${ }^{5}$ However, although the large-scale manufacture and use of polyethylene insulation for HVDC cables has been adopted for decades, one of the main reasons for the limited use of metal oxide nanoparticles is the poor understanding of the origin of the improved insulating properties. ${ }^{6-8}$ The particular interest in nanoparticles stem from their ability to create a very large particle-polymer interface at low particle fractions, which has been emphasized as the key to the significantly improved insulation properties. ${ }^{9-11}$ On one hand, the $10-30 \mathrm{~nm}$ particles provide a large particle-polymer interface/interphase in the composites that can favorably trap charge carriers, provided that agglomerates can be avoided that show more space charge accumulation within the composites. ${ }^{6,12}$ On the other, robust and selective surface modification reactions that ensure uniquely uniform coating of individual nanoparticles are scarce, which raises questions regarding the use of nanoparticles in large-scale HVDC applications since the agglomeration may result in cable insulation failure, with e.g. costly consequences. ${ }^{13,14}$ 
Micro-sized zinc oxide ( $\mathrm{ZnO})$ particles with porous hierarchical morphologies as filler in LDPE have been reported to yield a DC conductivity improvement similar to that achieved with nanoparticles, although the inter-particle distance (IPD) was significantly larger. ${ }^{1}$ An apparent benefit with the larger particle size is the limited agglomeration of the particles during processing, which results in reproducible particle dispersions. The theoretical values of the inter-particle center-to-center distance of a LDPE composite with $3 \mathrm{wt} \%$ spherical $\mathrm{ZnO}$ particles, if assuming optimal dispersion represented by a face-centered cubic lattice, can be calculated to increase from $160 \mathrm{~nm}$ to $5.3 \mu \mathrm{m}$ when increasing the particle size from $30 \mathrm{~nm}$ to $1 \mu \mathrm{m} .{ }^{15,16}$ This dramatically decreases the probability of having particle agglomeration. Interestingly, the larger $\mathrm{ZnO}$ particles also opens up for investigating how the conductive properties of the composites depend on the specific atomic terminations that become more defined as the $\mathrm{ZnO}$ enters the micrometer range dimensions. ${ }^{17}$

Previously, we have demonstrated that micro-sized $\mathrm{ZnO}$ particles with a variety of sizes, morphologies, surface areas and atomic terminations can be synthesized in a controlled manner giving systematic and reproducible particle characteristics, as employed in HVDC insulating composites. ${ }^{6,17-21}$ In the present work, the atomic terminations of micro-sized $\mathrm{ZnO}$ particles in LDPE composites are evaluated in terms of their influence on the DC conductivity, compared to that of nano-sized particles. The difference between micro-sized rod-shaped and porous ball-shaped particles, consisting of interconnected sheet morphologies, lies in the amount of zinc versus oxygen atoms exposed as particle surface terminations. ${ }^{20}$ The amount of exposed oxygen varies from $17 \%$ to $67 \%$ oxygen depending on the $\mathrm{ZnO}$ crystal shapes. ${ }^{17}$ We report to which extent atomic crystal terminations affect the conductivity of composites developed as insulation materials. The micro-sized particles were further encapsulated inside silica or a silsesquioxane coating with aliphatic tails, in order to be able to compare differently shaped particles with the same surfaces. The results reveal that the atomic surface terminations play an important and dominant role as a functional interface that determines the overall electrical properties of these materials. The results will consequently have direct implications on the development of particle composite materials envisioned useful in the next generation of HVDC cables, targeting transmissions voltages at $>1 \mathrm{MV}^{22}$

\section{Experimental}

\subsection{Materials}

2-Propanol ( $\geq 99.5 \%$, VWR), $n$-heptane ( $\geq 99 \%$, VWR), $p$-xylene ( $\geq 99 \%$, Sigma Aldrich), ammonium hydroxide (28-30\%, SigmaAldrich), ethanol, ( $\geq 96 \%$, VWR), Irganox 1076 (Ciba Specialty Chemicals), MilliQ water ("Type 1", following ISO 3696 and ASTM D1193-91, $18.2 \mathrm{M} \Omega \mathrm{cm}$ at $\left.25{ }^{\circ} \mathrm{C}\right)$, octyltriethoxysilane (C8, $\geq 98 \%$, Sigma-Aldrich), orthophosphoric acid (85\%, VWR), potassium permanganate $(\geq 99 \%$, VWR), sodium hydroxide $(\mathrm{NaOH}$, $\geq 98 \%$, Sigma-Aldrich), sulfuric acid (95-98\%, Sigma Aldrich), tetraethoxysilane ( $\geq 98 \%$, Sigma-Aldrich), zinc acetate dihydrate $\left(\mathrm{Zn}\left(\mathrm{CH}_{3} \mathrm{COO}\right)_{2} \cdot 2 \mathrm{H}_{2} \mathrm{O}, \geq 99 \%\right.$, Sigma Aldrich) and zinc nitrate hexahydrate $\left(\mathrm{Zn}\left(\mathrm{NO}_{3}\right)_{2} \cdot 6 \mathrm{H}_{2} \mathrm{O}, \geq 98 \%\right.$, Sigma-Aldrich) were used as received. Low-density polyethylene (LDPE) was provided by Borealis.

\subsection{Synthesis of $\mathrm{ZnO}$ particles}

Ball-shaped (BS) and rod-shaped (RS) ZnO particles were synthesized at 60 and $80{ }^{\circ} \mathrm{C}$, respectively, by adding an aqueous $\mathrm{NaOH}$ solution (at room temperature) to an aqueous zinc nitrate solution pre-heated to the synthesis temperature. For ball- and rod-shaped particles, 1 and $4 \mathrm{M} \mathrm{NaOH}$ aqueous solutions (250 mL), respectively, were used, and a $0.067 \mathrm{M}$ zinc nitrate aqueous solution $(750 \mathrm{~mL})$ was used for both particles. ZnO nanoparticles were synthesized by adding a $0.5 \mathrm{M} \mathrm{NaOH}$ aqueous solution (500 mL) pre-heated to $60{ }^{\circ} \mathrm{C}$ to a $0.2 \mathrm{M}$ zinc acetate aqueous solution $(500 \mathrm{~mL})$ at $60{ }^{\circ} \mathrm{C}$. The reactions were continued for $1 \mathrm{~h}$ under vigorous stirring. The synthesized particles were centrifuged thrice with intermediate changes of the solvent to MilliQ water followed by ultrasonication (2510-DTH, Branson) and thorough shaking. The cleaned particles were dried at $80{ }^{\circ} \mathrm{C}$ overnight, ground to a powder and finally dried at $80{ }^{\circ} \mathrm{C}$ overnight under reduced pressure (Vacucell, MMM Group).

\subsection{Post treatment of synthesized particles}

The 'as-synthesized' ball- and rod-shaped ZnO particles were either heat-treated or surface modified (except for direct implementation of unmodified particles in composites). Heat treatment of the synthesized particles was performed in an oven (H14-GAXP furnace, Micropyretic Heaters International Inc.) at $400{ }^{\circ} \mathrm{C}$ for $1 \mathrm{~h}$ in air. Silsesquioxane and silica coatings to modify the surface of the synthesized particles were obtained using octyltriethoxysilane and tetraethoxysilane. The particles were dispersed in MilliQ water by ultrasonication prior to the addition of 2-propanol. Ammonia and the silane were then added during vigorous stirring and the reaction was allowed to continue for $24 \mathrm{~h}$. The amounts of chemicals used for different surface treatments are presented in Table 1. After all the reactions, the modified particles were washed thrice by centrifugation in ethanol prior to drying at $80{ }^{\circ} \mathrm{C}$ overnight, grinding to a powder and further drying at $80{ }^{\circ} \mathrm{C}$ overnight under reduced pressure (Vacucell, MMM Group).

\subsection{Particle characterization}

The morphology of the synthesized ZnO particles was studied with a field emission scanning electron microscope (SEM,

Table 1 Amounts of chemicals used during surface treatment of $\mathrm{ZnO}$ particles

\begin{tabular}{|c|c|c|c|c|c|}
\hline $\begin{array}{l}\text { Particle } \\
\text { coating }\end{array}$ & $\begin{array}{l}\text { Particles } \\
\text { (g) }\end{array}$ & $\begin{array}{l}\text { Water } \\
(\mathrm{mL})\end{array}$ & $\begin{array}{l}\text { 2-Propanol } \\
(\mathrm{mL})\end{array}$ & $\begin{array}{l}\text { Ammonium } \\
\text { hydroxide }(\mathrm{mL})\end{array}$ & $\begin{array}{l}\text { Silane } \\
(\mathrm{mL})\end{array}$ \\
\hline $\mathrm{BS}-\mathrm{SiO}_{2}$ & 0.6 & 40.8 & 188 & 0.39 & 0.42 \\
\hline BS-C8 & 0.6 & 40.8 & 188 & 2.50 & 2.70 \\
\hline $\mathrm{RS}^{-\mathrm{SiO}_{2}}$ & 0.6 & 40.8 & 188 & 0.23 & 0.25 \\
\hline RS-C8 & 0.6 & 40.8 & 188 & 1.50 & 1.60 \\
\hline NP-SiO ${ }_{2}$ & 0.6 & 40.8 & 188 & 0.80 & 0.85 \\
\hline NP-C8 & 0.6 & 40.8 & 188 & 5.10 & 5.40 \\
\hline
\end{tabular}


Hitachi S-4800) after sputtering (Cressington $208 \mathrm{HR}$ ) with a current of $80 \mathrm{~mA}$ and $\mathrm{Pt} / \mathrm{Pd}$ for 20 seconds to form a conductive layer. An acceleration voltage of $5 \mathrm{kV}$ and emission current of $10 \mu \mathrm{A}$ were used for the SEM microscopy of the ZnO particles. The size distributions of the synthesized particles and the thickness of the surface functionalization treatments were assessed by transmission electron microscopy (TEM, HT7700, Hitachi) using an acceleration voltage of $100 \mathrm{kV}$, and the diameter of ball-shaped particles and the length of rodshaped particles from the TEM micrographs were determined using Image (National Institute of Health, Maryland, USA). The specific surface area (SSA) of the $\mathrm{ZnO}$ particles was determined by the BET (Brunauer-Emmett-Teller) method using nitrogen adsorption/desorption measurements performed in a Micromeritics ASAP 2000 at $77 \mathrm{~K}$. Thermogravimetric analysis (TGA, Mettler Toledo TGA/DSC1) was used to measure the mass loss of $\mathrm{ZnO}$ particles by placing $10 \pm 1 \mathrm{mg}$ in $70 \mu \mathrm{L}$ alumina crucibles followed by pre-drying in the instrument by ramping to $140{ }^{\circ} \mathrm{C}$ in nitrogen prior to heating from 30 to $800{ }^{\circ} \mathrm{C}$ in oxygen. The rate of temperature change was $10{ }^{\circ} \mathrm{C} \min ^{-1}$ and the gas flow was $50 \mathrm{~mL} \mathrm{~min}^{-1}$. The molecular structure of the particle coatings was characterized by Fourier-transform infrared spectroscopy (FTIR, Spectrum 100, PerkinElmer) with a Golden Gate accessory (Graseby Specac LTD). X-ray diffraction (XRD, ARL X'TRA, Thermo) with a step size of $0.02^{\circ}, 45 \mathrm{kV}$ and $40 \mathrm{~mA}$ and $\mathrm{Cu} \mathrm{K} \alpha$ radiation was used to investigate the crystal structure and purity of the synthesized particles. Material studio (version 4.3, Accelrys Inc., 2007) was used to illustrate the dominating crystal faces of the synthesized $\mathrm{ZnO}$ particles.

\subsection{Composite characterization}

Differential scanning calorimetry (DSC, TG/DSC1, Mettler Toledo) was used to obtain the mass crystallinity, melting and crystallization temperatures. A piece of the compressionmolded specimen ( $5 \pm 0.5 \mathrm{mg}$ ) was placed in a $40 \mu \mathrm{L}$ aluminum cup and was measured in nitrogen $\left(50 \mathrm{~mL} \mathrm{~min}^{-1}\right.$ flow rate) with a heating rate of $10{ }^{\circ} \mathrm{C} \mathrm{min}^{-1}$. The DSC method consisted of cooling to $-50{ }^{\circ} \mathrm{C}$, heating to $200{ }^{\circ} \mathrm{C}$, cooling to $-50{ }^{\circ} \mathrm{C}$ and finally heating to $200{ }^{\circ} \mathrm{C}$ with a $5 \mathrm{~min}$ isothermal step between each temperature change. The mass crystallinity was calculated according to the total enthalpy method: ${ }^{23}$

$$
w_{\mathrm{c}}=\frac{\Delta h_{\mathrm{f}}}{\Delta h_{\mathrm{f}}^{0}-\int_{T_{1}}^{T_{\mathrm{m}}^{0}}\left(c_{\mathrm{p}, \mathrm{a}}-c_{\mathrm{p}, \mathrm{c}}\right) \mathrm{d} T} \times 100
$$

where $w_{\mathrm{c}}$ is the mass crystallinity, $T_{\mathrm{m}}^{0}$ the equilibrium melting point of polyethylene (PE), $\Delta h_{\mathrm{f}}$ the enthalpy of melting, $\Delta h_{\mathrm{f}}^{0}$ the melting enthalpy for $100 \%$ crystalline $\mathrm{PE}$ at $T_{\mathrm{m}}^{0}$, and $c_{\mathrm{p}, \mathrm{c}}$ and $c_{\mathrm{p}, \mathrm{a}}$ are, respectively, the heat capacities for the crystalline and amorphous components. Data for $T_{\mathrm{m}}^{0}(414.6 \mathrm{~K}), \Delta h_{\mathrm{f}}^{0}\left(293 \mathrm{~J} \mathrm{~g}^{-1}\right)$ and heat capacities for PE were obtained from Wunderlich and Bauer. $^{24,25}$ SEM was used to assess the dispersion of $\mathrm{ZnO}$ particles in the LDPE composites using the back-scattering detector for enhanced contrast between the LDPE matrix and $\mathrm{ZnO}$ particles. The composites were freeze-cracked in liquid nitrogen, coated by sputtering and investigated using an acceleration voltage of $5 \mathrm{kV}$ and an emission current of $10 \mu \mathrm{A}$. The average inter-particle distance (IPD) was obtained from the micrographs by marking the particles and calculating the particle/agglomerate positions according to their centers of gravity and their respective nearest neighbor distances. Etching of freeze-cracked cross-sections was performed to investigate the crystal polymer morphology using SEM. The samples were etched for $2 \mathrm{~h}$ in an etchant consisting of $4 \mathrm{~mL}$ water, $16 \mathrm{~mL}$ orthophosphoric acid, $40 \mathrm{~mL}$ sulfuric acid and $600 \mathrm{mg}$ potassium permanganate. The samples were rinsed by water and dried in a desiccator overnight after the etching procedure.

\subsection{Composite preparation}

The LDPE composites with $\mathrm{ZnO}$ particles were prepared by dispersing the particles ( $3 \mathrm{wt} \%$ in the composite) in $3 \mathrm{~mL}$ heptane using ultrasonication (2510-DTH, Branson) followed by the addition of the antioxidant Irganox 1076 (0.02 wt\% in the composite). LDPE pellets were ground after quenching in liquid nitrogen (Retsch ZM 200 with $12000 \mathrm{rpm}$ ) and the powder was added to the particle dispersion followed by shaking of the mixture using a Vortex Genie 2 Shaker (G560E, Scientific Industries) for $1 \mathrm{~h}$ and finally drying overnight at $80{ }^{\circ} \mathrm{C}$. The dried mixture was further shaken for $30 \mathrm{~min}$ and then extruded (Micro 5cc Twin Screw Compounder, DSM Xplore) with $100 \mathrm{rpm}$ at $150{ }^{\circ} \mathrm{C}$ for $6 \mathrm{~min}$ and finally vacuum dried at $80{ }^{\circ} \mathrm{C}$ overnight (Vacucell, MMM Group) prior to compression molding (LabPro 400 press, Frontlijne Grotnes). The pellets were placed in the middle of the mold with polyethylene terephthalate as a protective film on both sides of the sample. The temperature was increased to $130{ }^{\circ} \mathrm{C}$ and a contact pressure was applied for $10 \mathrm{~min}$, followed by pressing with a high force $(200 \mathrm{kN})$ at $130{ }^{\circ} \mathrm{C}$ for $10 \mathrm{~min}$ prior to cooling to room temperature under the same force for $6 \mathrm{~min}$.

\subsection{Electrical characterization}

The compression-molded samples $(0.3 \mathrm{~mm}$ thick and $75 \mathrm{~mm}$ in diameter) were characterized by HVDC leakage current measurements and the apparent conductivity $(\sigma)$ was calculated according to the equation:

$$
\sigma=\frac{I \cdot d}{U \cdot A}
$$

where $I$ is the measured current, $d$ is the thickness of the sample, $U$ is the applied DC voltage and $A$ is the area of the sensing electrode. ${ }^{26}$ The DC conductivity was calculated from the current after $15 \mathrm{~h}$ of polarization. The measurement setup consisted of a high voltage supply (HCP 35-12500, FUG), electrometer (6517B, Keithley), oven (FED 115, Binder), protection for the electrometer and a CPU with a LabVIEW software to obtain the measurement data. A three-electrode system was used with a sensing electrode (30 $\mathrm{mm}$ in diameter), a guard electrode of brass and a high voltage electrode of stainless steel with a film of Powersil 440 placed on the electrode to give good contact with the PE sample. A voltage of $9 \mathrm{kV}$ was applied corresponding to an electric field of $30 \mathrm{kV} \mathrm{mm}^{-1}$. Each sample was measured only once to eliminate any induced effects 
possible arising from having the samples exposed to the high electrical fields and the resulting thermal exposure.

Surface potential measurements were performed using an atomic force microscope (Bruker Dimension Icon) in the intermodulation electrostatic force microscopy (ImEFM) mode. ${ }^{27}$ A cantilever with platinum coating on the tip side was mechanically oscillated to an amplitude of roughly $80 \mathrm{~nm}$ at a frequency $\omega_{\mathrm{D}}$, near its resonance frequency (MicroMasch HQ:XSC11-C/Pt, nominal resonance frequency $155 \mathrm{kHz}$, nominal stiffness $7 \mathrm{~N} \mathrm{~m}^{-1}$ ). Simultaneously a voltage was applied between the tip and the surface consisting of an AC oscillation, $6 \mathrm{~V}$ at $\omega_{\mathrm{E}}=2 \pi 500 \mathrm{~Hz}$, and optionally a DC bias. Due to these two drive tones the nonlinear electrostatic force between the tip and the surface

$$
F_{\mathrm{EL}}=\frac{1}{2} \frac{\partial C}{\partial z}\left(V_{\mathrm{CPD}}+V_{\mathrm{AC}} \cos \left(\omega_{\mathrm{E}} t+\phi_{\mathrm{E}}\right)\right)^{2}
$$

will create four different "side-bands" or intermodulation products at the sum and difference frequencies $\omega_{\mathrm{D}} \pm \omega_{\mathrm{E}}$ and $\omega_{\mathrm{D}} \pm 2 \omega_{\mathrm{E}}$. The amplitude and phase of these oscillations depend on the applied AC voltage, $V_{\mathrm{AC}}$ and the DC contact potential difference between the tip and the sample, $V_{\mathrm{CPD}}$, which in turn depend on both the inherent contact potential between the materials as well as the applied DC bias. By measuring both amplitude and phase of the intermodulation products one can solve for the contact potential difference

$$
V_{\mathrm{CPD}}=\frac{V_{\mathrm{AC}}}{4} \frac{\hat{F}_{\omega_{\mathrm{D}} \pm \omega_{\mathrm{E}}}}{\hat{F}_{\omega_{\mathrm{D}} \pm 2 \omega_{\mathrm{E}}}} \mathrm{e}^{i \phi_{\mathrm{E}}}
$$

See Borgani et $a .^{27}$ for full derivation. The complex force components $\hat{F}_{\omega}$ were calculated from the complex amplitudes of the motion $\hat{z}_{\omega}$ by accounting for the transfer function of the cantilever. Motion was measured using a multifrequency lockin amplifier (Intermodulation Products $\mathrm{AB}$ ). Surfaces were imaged with multiple DC bias applied while recording the resulting $V_{\mathrm{CPD}}$. This method has previously been suggested to demonstrate local charging injection on nanoparticles embedded in LDPE. $^{28}$

\section{Results}

\subsection{Preparation of $\mathrm{ZnO}$ particles for nanodielectric composites}

Fig. 1 shows micrographs of the ball- and rod-shaped $\mathrm{ZnO}$ particles synthesized in the absence of surface modification, i.e. by tuning the reactant ratio and temperature during the synthesis to favor the directional growth into the morphologies obtained. ${ }^{18}$ The ball-shaped particles with a diameter of ca. $1.5 \mu \mathrm{m}$ consisted of a hierarchical organization of interconnected sheets with an average thickness of $c a .25 \mathrm{~nm}$ (Fig. 1a-c). ${ }^{20}$ The dominant surfaces of the sheets correspond to the $\{2 \overline{1} \overline{1} 0\}$ crystal planes with a majority of oxygen terminations $(67 \%)$, which was established by atomistic modeling (see Fig. S1a, ESI $\dagger) .{ }^{17}$ Fig. 1d-f shows the rod-shaped ZnO particles with an average length of $c a .1 \mu \mathrm{m}$ and width of $c a .50 \mathrm{~nm}$ (an aspect ratio of 20) that were formed by growth in the c-direction. ${ }^{21}$ Atomistic modeling of the facetted planes along the hexagonal rods indicated that the total amount of oxygen terminations was smaller $(17 \%)$, i.e. from the (1100), (1010) and (0110) crystal planes (Fig. S1b, ESI $\dagger$ ). ${ }^{17}$

Fig. 1c and $f$ show the morphology of the nanosheets in the ball-shaped and the rod-shaped particles after heat treatment

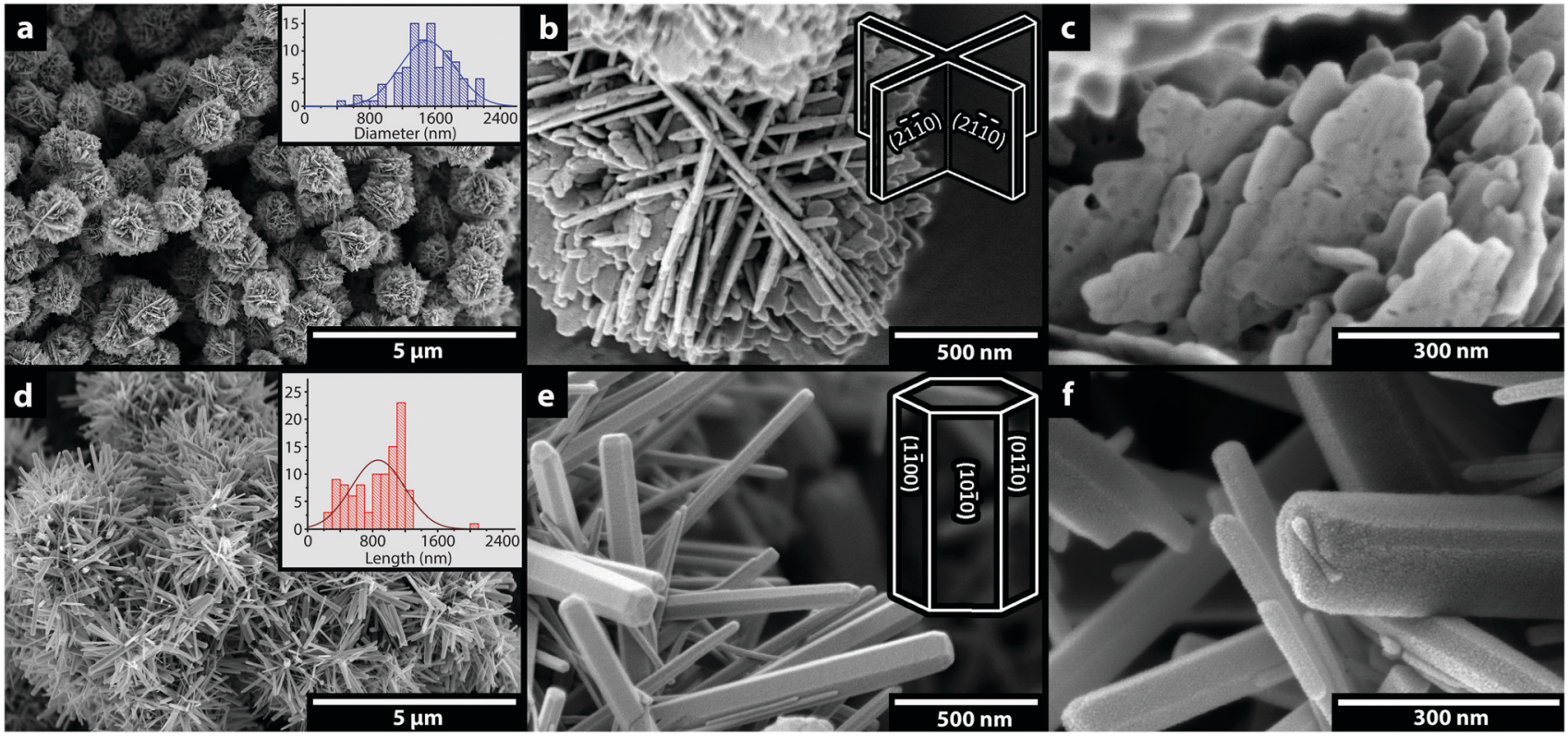

Fig. 1 Scanning electron micrographs of particles with different $\mathrm{Zn} / \mathrm{O}$ surface terminations. Ball-shaped particles are shown in (a-c), where (c) shows the particles after $400{ }^{\circ} \mathrm{C}$ heat treatment. Rod-shaped particles are shown in (d-f), where (f) shows particles after $400{ }^{\circ} \mathrm{C}$ heat treatment. The insets in (a) and (d) show the size distributions of the particles, and the dominant crystal planes for ball- and rod-shaped particles are shown as insets in (b) and (e), respectively. 
at $400{ }^{\circ} \mathrm{C}(1 \mathrm{~h}$, air). The formation of $5-10 \mathrm{~nm}$ "potholes" is visible in the sheets as the crystal surface collapsed due to imperfections associated with dominant growth in the $\langle 01 \overline{1} 0\rangle$ and [0001] directions, whereas the rods grown in their $c$-axis showed perfectly smooth surfaces. ${ }^{20,29,30}$ The specific surface area (SSA) was reduced by the heat treatment from 16.7 to $13 \mathrm{~m}^{2} \mathrm{~g}^{-1}$ for the balls and from 9.9 to $9.3 \mathrm{~m}^{2} \mathrm{~g}^{-1}$ for the rods. ${ }^{20}$ This relatively small reduction is commonly observed with the elimination of porosity at a temperature of $400{ }^{\circ} \mathrm{C}$, a temperature that allows solitary particle annealing while preventing the inter-particle growth that develops at higher temperatures $\left(>600{ }^{\circ} \mathrm{C}\right) .{ }^{19-21}$

\subsection{Effect of $\mathrm{ZnO}$ particle morphology/terminations on the DC conductivity}

Fig. 2 shows the apparent DC conductivity at $60{ }^{\circ} \mathrm{C}$ and $30 \mathrm{kV} \mathrm{mm}^{-1}$ of composites containing $3 \mathrm{wt} \%$ of unmodified ball- and rod-shaped ZnO particles compared to that of pristine LDPE. The charging current of the composites dropped markedly during the first $10 \mathrm{~s}$ for both composites resulting in apparent conductivities $c a .2$ orders of magnitude lower than that of the reference LDPE $\left(1 \times 10^{-14}\right.$ and $1 \times$ $10^{-12} \mathrm{~S} \mathrm{~m}^{-1}$, respectively). This difference between the composites and the reference LDPE also prevailed at longer times $(15 \mathrm{~h})$, with a continuously decreasing current, which is consistent with previous reports in the literature. ${ }^{26}$

A marked difference in conductivity between the composites containing the ball- (BS) and rod-shaped (RS) particles was however always observed after $15 \mathrm{~h}$ (54000 s); see the inset in Fig. 2. This difference corresponded to a current twice as high passing through the composites containing the ball-shaped particles with its extensive oxygen-terminated surfaces, i.e. $2.4 \times 10^{-16} \mathrm{~S} \mathrm{~m}^{-1}$. Note that the curves for the composites separate already after 200 seconds.

\subsection{Influence of heat treatment and atomic terminations of ZnO particles on the DC conductivity}

The ball- and rod-shaped particles were further heated-treated at $400{ }^{\circ} \mathrm{C}(1 \mathrm{~h}$, air $)$ to refine their crystal faces, allowing for

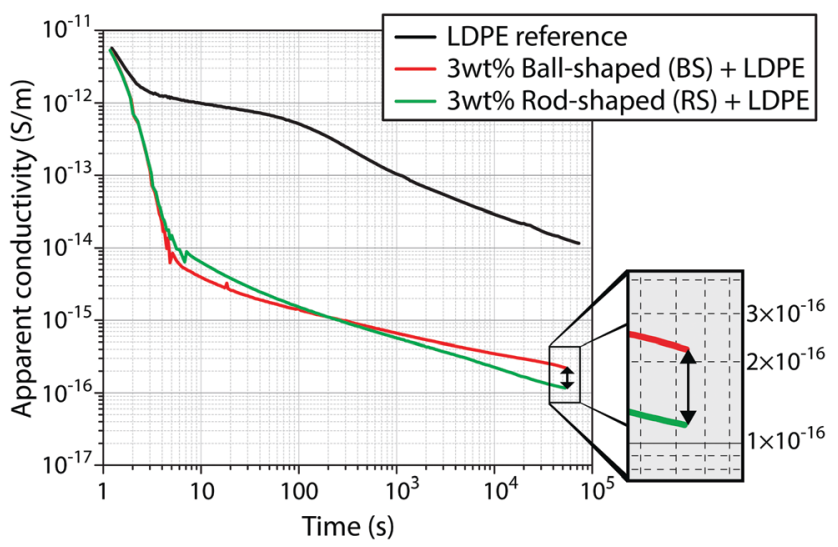

Fig. 2 Apparent conductivity of composites containing 3 wt\% ball- or rod-shaped particles and of pristine LDPE at $30 \mathrm{kV} \mathrm{mm}^{-1}$ at $60{ }^{\circ} \mathrm{C}$. Several measurements up to 2 days were made independently on separately prepared samples, see Fig. S2 (ESI†). atomic rearrangement in the outermost layers of the particles. This heat treatment also eliminated the surface-located hydroxyl groups on the zinc oxide crystals by condensation. ${ }^{20}$ Fig. 3 shows the apparent conductivity at $60{ }^{\circ} \mathrm{C}$ and $30 \mathrm{kV} \mathrm{mm}^{-1}$ for LDPE composites containing $3 \mathrm{wt} \%$ of heat-treated ball- and rodshaped $\mathrm{ZnO}$ particles (dashed lines), compared to the same particles without thermal treatment (solid lines).

The conductivity of the heat-treated ball-shaped particles was twice as high after $15 \mathrm{~h}$ as that of the composite containing ball-shaped particles without heat treatment (red lines, Fig. 3). A smaller increase in conductivity was observed in the case of the composite containing heat-treated rod-shaped particles compared with the same particles without heat treatment (green lines, Fig. 3). Both the composites containing heattreated particles showed a higher conductivity when more refined crystal faces of the $\mathrm{ZnO}$ crystals were exposed to the polymer matrix (with eliminated hydroxyl groups). It was also noted that the slopes of the curves for the composite containing heat-treated rod-shaped particles resembled the slope of the LDPE reference, i.e. $n \approx 0.5$ in the power law function $I \approx t^{-n}$, whereas the slope for the composites containing ball-shaped particles leveled out at longer polarization times (see the extrapolation in Fig. S4, ESI $\dagger$ ). ${ }^{26}$ The different inclinations of the curves in the direction of the PE reference, showing the charging current over time, may be related to the ability of the composite to disseminate/distribute charges over the atomic surfaces inside the composites.

Fig. $4 \mathrm{a}$ and $\mathrm{b}$ show the mass loss $\left(30-800{ }^{\circ} \mathrm{C}\right)$ of the dry particles immediately after synthesis, and their mass loss after a heat treatment at $400{ }^{\circ} \mathrm{C}$ for 1 hour. The initial mass loss (30-185 ${ }^{\circ} \mathrm{C}$ ) was due to desorption of loosely bonded water and the mass loss at temperatures above $185{ }^{\circ} \mathrm{C}$ is attributed to the condensation of surface located hydroxyl groups. ${ }^{20}$ The greater mass loss for the ball-shaped particles $\left(m_{\text {ball }}=1.5 \%\right.$ compared to $m_{\text {rod }}=0.7 \%$, Fig. $4 \mathrm{a}$ and b) was associated with their larger

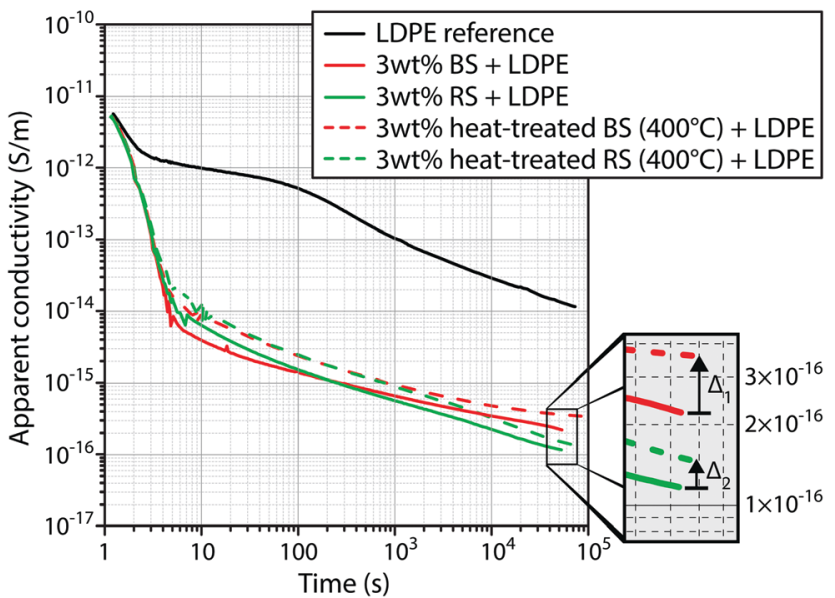

Fig. 3 Apparent conductivity at $60{ }^{\circ} \mathrm{C}$ with an electric field of $30 \mathrm{kV} \mathrm{mm} \mathrm{m}^{-1}$ for ZnO LDPE composites containing $3 \mathrm{wt} \%$ unmodified or heat-treated $\left(400{ }^{\circ} \mathrm{C}\right)$ particles in the shape of rods or balls. The reproducibility of measurements on the composites containing heat-treated particles is shown in Fig. S3 (ESI†). 

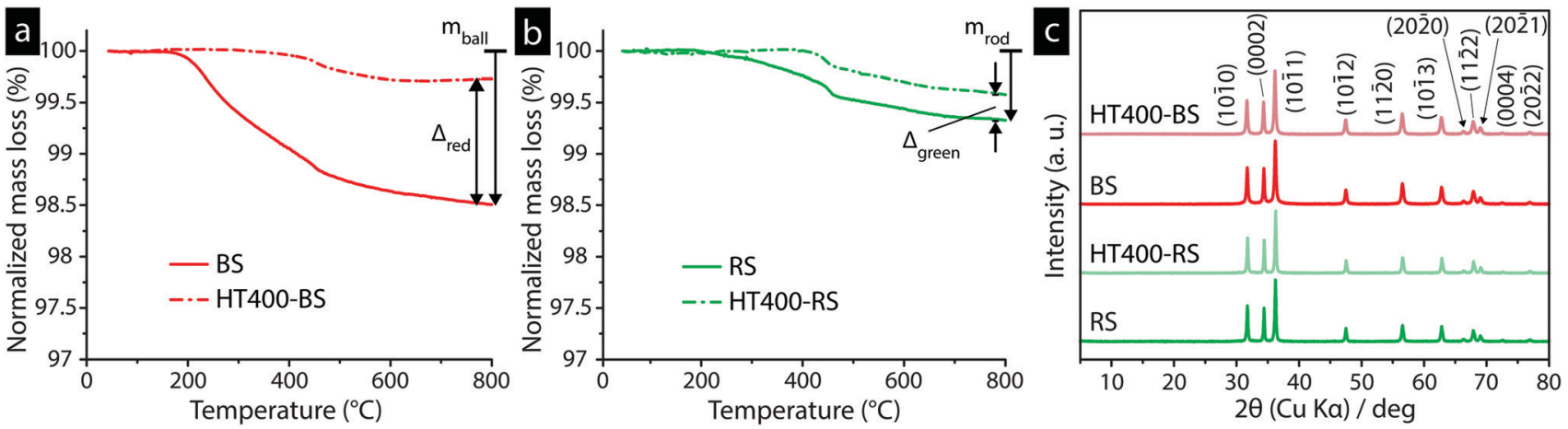

Fig. 4 Characterization of unmodified and heat-treated ball- and rod-shaped ZnO particles by ( $a$ and b) thermogravimetry and (c) X-ray diffraction.

surface area and the greater amount of hydroxyl groups present on the oxygen-terminated surfaces. The mass losses observed in the gravimetric test (TGA) corresponded to the loss of a ca. 4 times larger amount of hydroxyl groups from the ballshaped particles $\left(\Delta_{\text {red }}=1.2 \%\right.$ compared to $\left.\Delta_{\text {green }}=0.3 \%\right)$ during their exposure at $400{ }^{\circ} \mathrm{C}$. In essence, the $400{ }^{\circ} \mathrm{C}$ treatment of the particles resulted in a more accurate representation of the modelled oxygen concentration of $67 \%$ for sheets and $17 \%$ for rods, reflecting a $c a$. 6 times higher oxygen concentration facing the polymer matrix for an equivalent mass of ball- and rod-shaped particles in the composites. The difference in conductivity $\left(\Delta_{1}>\Delta_{2}\right.$ in Fig. 3$)$ of the composites therefore corresponded to the frequency of oxygen terminations in the heat-treated particles.

At the same time it could be concluded that a small amount of hydrogen atoms present in hydroxyl groups (present as natural on the surface of the particles) appears to be beneficial for the insulating properties, whereas a surface refinement at even more elevated temperatures $\left(>400{ }^{\circ} \mathrm{C}\right)$ was not possible without significantly affecting the surface area, morphology and causing inter-particle growth. ${ }^{20,21}$ It should here be noted that although the $\mathrm{ZnO}$ crystals contain a number of mixed terminations at the crystal edges, the dominant nature of the surfaces is still present. Fig. 4c shows XRD diffractograms for unmodified and heat-treated $\left(400{ }^{\circ} \mathrm{C}\right)$ rod- and ball-shaped $\mathrm{ZnO}$ particles. Only peaks corresponding to the wurtzite hexagonal crystal structure with lattice parameters of $a=3.25 \AA$ and $c=5.21 \AA$ were present in agreement with the inorganic crystal structure database; collection \# 067849. The XRD data were also used to confirm that the heat treatment at $400{ }^{\circ} \mathrm{C}$ induced no crystal growth, which occurred for ball- and rod-shaped particles when heated above $600{ }^{\circ} \mathrm{C} .{ }^{20,21}$

\subsection{Elimination of zinc and oxygen particle terminations by silica coatings and their effect on the DC conductivity}

To confirm that the zinc or oxygen terminations in the $\mathrm{ZnO}$ particle surfaces significantly affected the DC conductivity of the composites, the rod- and ball-shaped particles were encapsulated with $5 \mathrm{~nm}$ amorphous silica coatings (Fig. $5 \mathrm{~b}$ and c). The conductivities were significantly higher already after ca. $10 \mathrm{~s}$ of polarization for the composite with the coated particles, and over time the current was always higher than that of the composites containing uncoated particles, see Fig. 5a. After $5.4 \times 10^{4} \mathrm{~s}$, the conductivity was almost one order of magnitude higher for the composites with silica-encapsulated particles. The embedded zinc and oxygen crystal terminations in the $\mathrm{ZnO}$ crystal faces could at this point be concluded to have no effect on the conductivity since the currents overlapped after the long polarization times. Accordingly, the micro-sized particles not only allowed making conclusions concerning the impact of their surface nature due to their exposed lattices but also allowed for making these conclusions since the dispersion of the larger particles in the polyethylene matrix were unaffected by detrimental agglomeration, which is further discussed in Section 3.6.

Infrared spectroscopy was used to confirm that a silica network was formed on the particle surfaces (Fig. 5d), leading to complete encapsulation of the metal oxide surface terminations. Fig. 5e shows that more water was condensed from the silica surfaces of the modified ball- and rod-shaped particles than from the unmodified particles $(1.9 \%$ compared to $1.2 \%$, respectively), which was consistent with the presence of more hydroxyl groups on the silica-coated particle surfaces. Any further optimization of the silica coating thicknesses' for the different particles was of minor relevance for the electrical data since the reported distance for electron tunneling is ca. 1-4 nm, which is shorter than the coating thickness. ${ }^{31-34}$

\subsection{The effect of aliphatic units in the silicone oxide coatings on the DC conductivity}

The marked effect of having oxygen present in the outer coating layer of the particles (Fig. 5a), increasing the current by an order of magnitude, was further investigated by coating the particles with the silica-related silsesquioxane coating. This provided a composition almost identical as that of the condensed silica coatings, while being terminated with hydrocarbon functional units consisting of 8 repetitive carbon atoms ( $c f$. molecular illustrations in the insets, Fig. 5a and 6a). Fig. 6a shows the electrical data for the particles coated with the amorphous silsesquioxane coating containing saturated hydrocarbon functional aliphatic units (C8). Not only did the eight carbon atoms eliminate the influence of the $\mathrm{ZnO}$ terminations and the oxygen in the silica coating, but they also suppressed the conductivity compared to that of the particles 
a

$3 w+\%$ reference

- $3 w t \%$ RS + LDPE $3 w \mathrm{wt} \% \mathrm{SiO}_{2}-\mathrm{RS}+\mathrm{LDPE}$

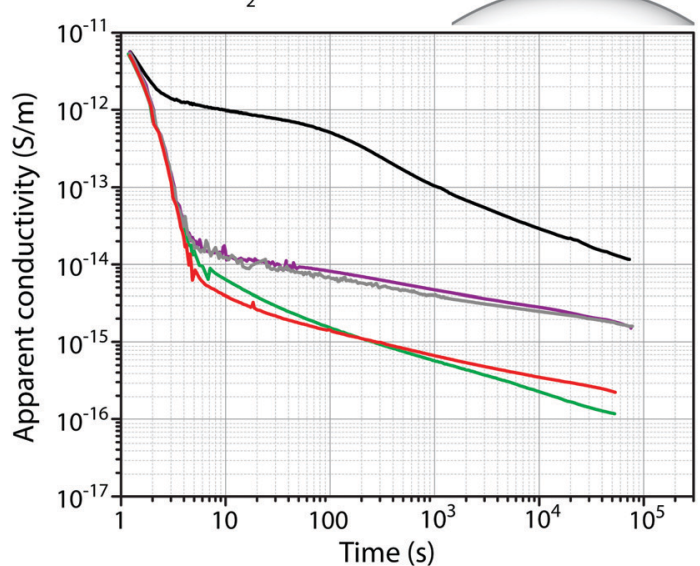

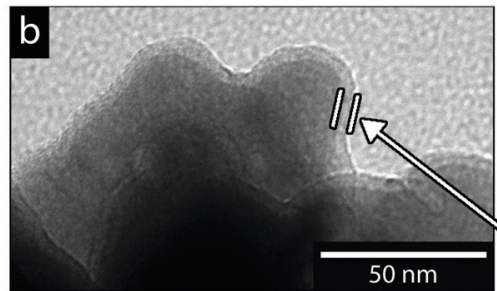
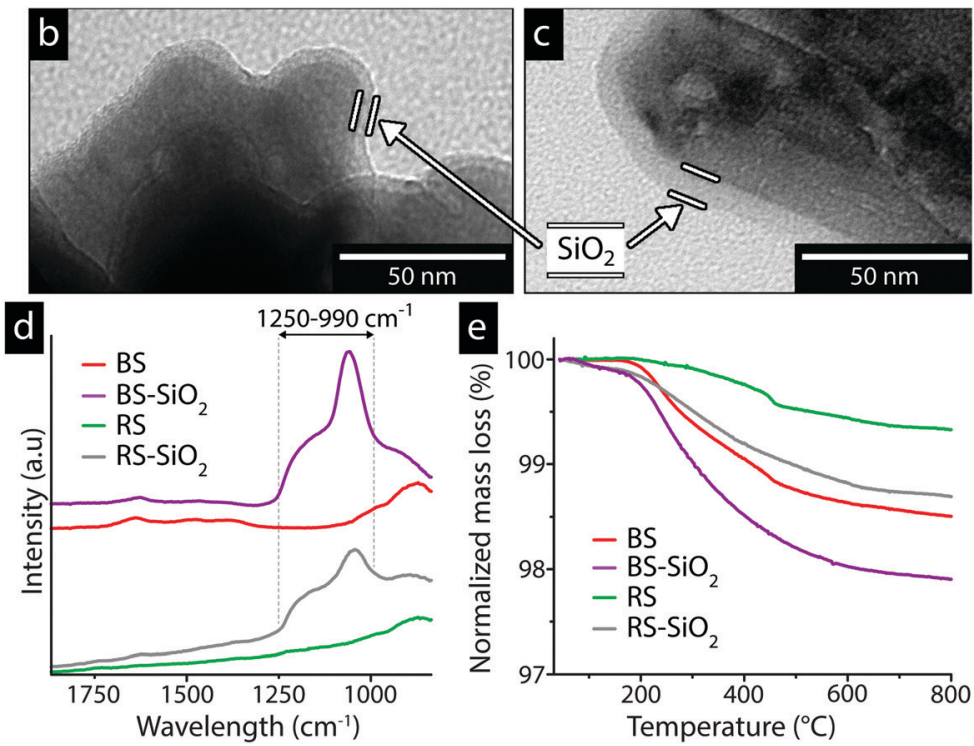

Fig. 5 (a) Apparent conductivity of LDPE composites containing $3 \mathrm{wt} \%$ silica modified ball- or rod-shaped $\mathrm{ZnO}$ particles measured at $60{ }^{\circ} \mathrm{C}$ and $30 \mathrm{kV}$ mm ${ }^{-1}$. The inset in (a) illustrates the molecular structure of the silica coating and silane monomer. Electron microscopy showing the coatings on (b) ball- and (c) rod-shaped particles, and (d) characterization of the 4-7 nm thick coatings by infrared spectroscopy and (e) thermogravimetry. The presence of silica was confirmed by the absorption band at $990-1250 \mathrm{~cm}^{-1}$ with the peak intensity at $1058 \mathrm{~cm}^{-1}$, corresponding to asymmetric Si-O-Si stretching vibrations. ${ }^{35-37}$ Table S1 (ESI $\dagger$ ) lists the measured conductivities, and additional micrographs of the unmodified particles are shown in Fig. S5 (ESI $\dagger$ ).

a
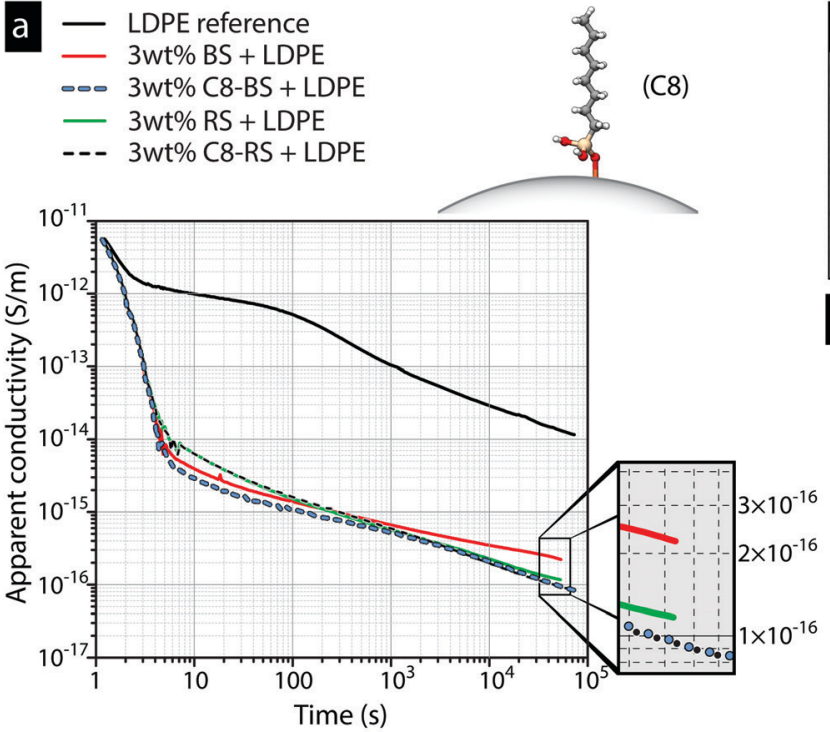

(C8)
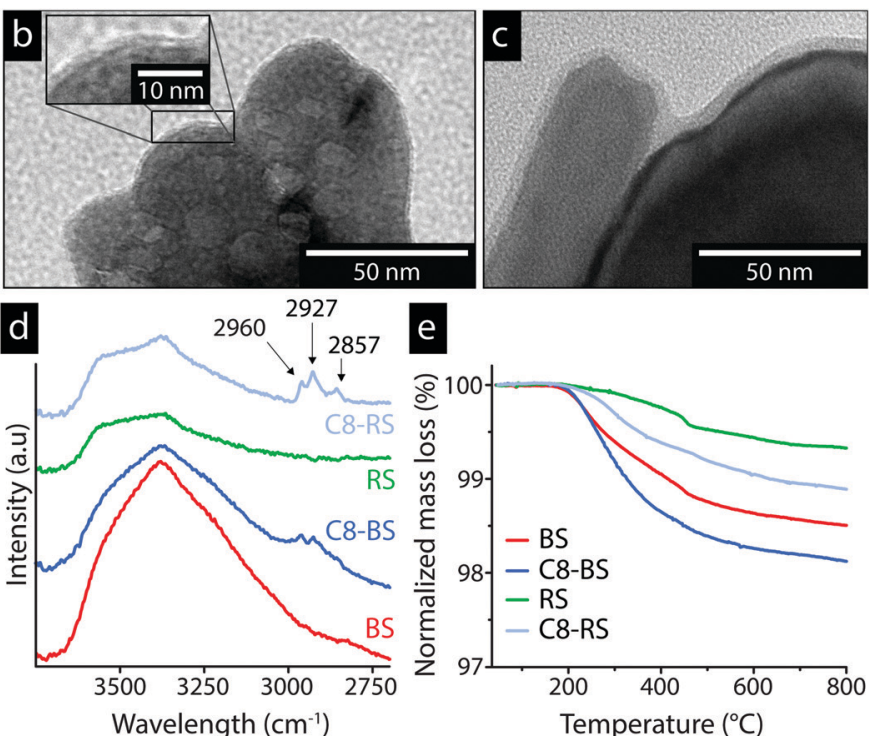

Fig. 6 (a) Apparent conductivity of LDPE composites containing $3 \mathrm{wt} \% \mathrm{C} 8$ modified ball- or rod-shaped ZnO particles measured at $60{ }^{\circ} \mathrm{C}$ and $30 \mathrm{kV} \mathrm{mm}^{-1}$. The inset in (a) illustrates the molecular structure of the C8 silsesquioxane bonded to the surface. The electron micrographs show (b) ballshaped particles with $2 \mathrm{~nm}$ coatings and (c) rod-shaped particles with $5 \mathrm{~nm}$ coatings. Characterization of the C8 functional silsesquioxane encapsulated particles by infrared spectroscopy is shown in (d) and by thermogravimetry in (e). The inset in (b) shows a magnification of the C 8 coating on ball-shaped particles. The presence of alkyl groups from the hydrocarbon tail of the silsesquioxane were confirmed by the peaks at $2800-3000 \mathrm{~cm}^{-1}$ assigned to the stretching vibrations of $\mathrm{C}-\mathrm{H}$ bonds. ${ }^{15,37}$ Table $\mathrm{S} 1(\mathrm{ESI}+)$ lists the measured conductivities, and additional micrographs of the unmodified particles are shown in Fig. S5 (ESI†).

without coatings. This lowering of the conductivity was most evident for composites with ball-shaped particles containing dominantly oxygen terminations. The inset in Fig. 6a shows that the composite conductivity of both the ball-shaped (C8-BS) and rod-shaped (C8-RS) particles was less than $10^{-16} \mathrm{~S} \mathrm{~m}^{-1}$ after $5.4 \times 10^{4} \mathrm{~s}(15 \mathrm{~h})$, with an almost complete overlap after ca. $20 \min \left(>10^{3} \mathrm{~s}\right)$.

Fig. $6 \mathrm{~b}$ and $\mathrm{c}$ show electron micrographs of C8 modified ball- and rod-shaped particles, and Fig. 6d shows the FTIR spectra of the coated particles confirming the carbon functionality by the 
peaks at around $2900 \mathrm{~cm}^{-1}$. The greater mass loss of the C8 modified ball-shaped particles than that of the rod-shaped particles (Fig. 6e), ca. $1.9 \%$ compared to $1.1 \%$, was due to the greater surface area of the former.

\subsection{The effects of coating surface functionality on the conductivity of $\mathrm{ZnO}$ nanoparticle composite systems}

Fig. 7 shows that the same trend was observed for the pyramidal shaped $\mathrm{ZnO}$ nanoparticles with mixed atomic terminations $(25 \mathrm{~nm}$, $34 \mathrm{~m}^{2} \mathrm{~g}^{-1}$ in SSA) as for the micro-sized particles. The conductivity of the composite containing unmodified $\mathrm{ZnO}$ nanoparticles was more than two orders of magnitude lower than that of the LDPE reference, while also significantly lower than that of composites with micro-sized $\mathrm{ZnO}$ particles (compare dashed black with dashed green line for the rod-shaped particles, see Fig. 7).

Fig. 7 shows that the C8 coating on the nanoparticles lowered the electrical conductivity from $3.5 \times 10^{-17}$ to $1 \times$ $10^{-17} \mathrm{~S} \mathrm{~m}^{-1}$. The $\mathrm{C} 8$ coating was therefore termed a low current stabilizing particle interface (LCSPI). Fig. 7 also shows that when the same nanoparticles were encapsulated with a $4 \mathrm{~nm}$ silica coating, a conductivity $c a$. one order of magnitude higher than that of the unmodified nanoparticles was observed. Table S1 (ESI $\dagger$ ) shows tabulated conductivities after $15 \mathrm{~h}$ polarization, and Fig. S6 (ESI $\dagger$ ) provides more information about the characterization of the nanoparticles.

Fig. 8 shows the dispersion of the unmodified $\mathrm{ZnO}$ particles in the polyethylene matrix at a $3 \mathrm{wt} \%$ concentration. Only solitary particles were observed for the largest $1.5 \mu \mathrm{m}$ ballshaped particles, as shown in Fig. 8a. This was also the dominantly observed particle distribution for the smaller $1 \mu \mathrm{m}$ rod-shaped particles, although an agglomerate several $\mu \mathrm{m}$ in size is shown at the top in Fig. 8b. The dispersion of the unmodified very high surface area nanoparticles was poor, the $25 \mathrm{~nm}$ particles being associated into larger agglomerates, Fig. 8c. A qualitative comparison of the particle sizes is presented in the micrograph insets in Fig. 8. Note that one or two particles are present in Fig. $8 \mathrm{a}$ and b, whereas the $5 \mu \mathrm{m}$

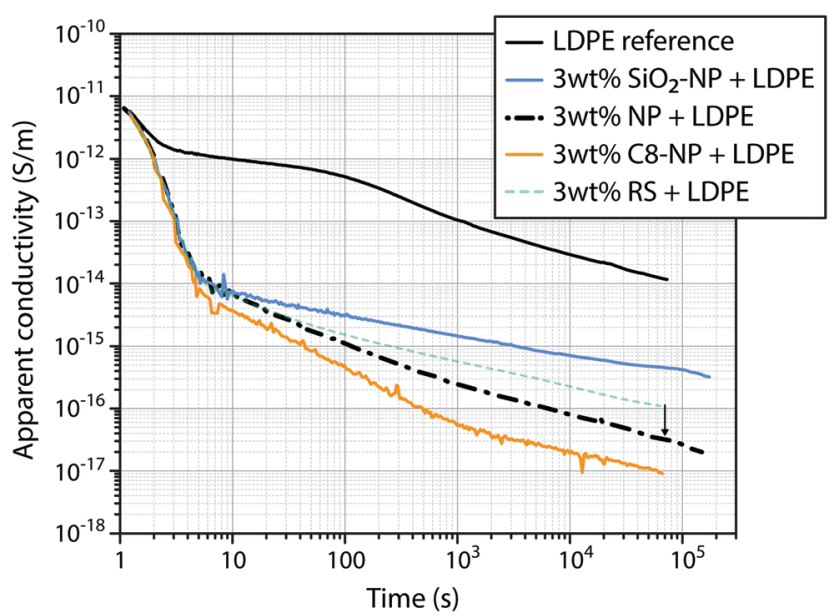

Fig. 7 The apparent conductivity at $60{ }^{\circ} \mathrm{C}$ and $30 \mathrm{kV} \mathrm{mm}{ }^{-1}$ for LDPE composites containing $3 \mathrm{wt} \% \mathrm{ZnO}$ nanoparticles.
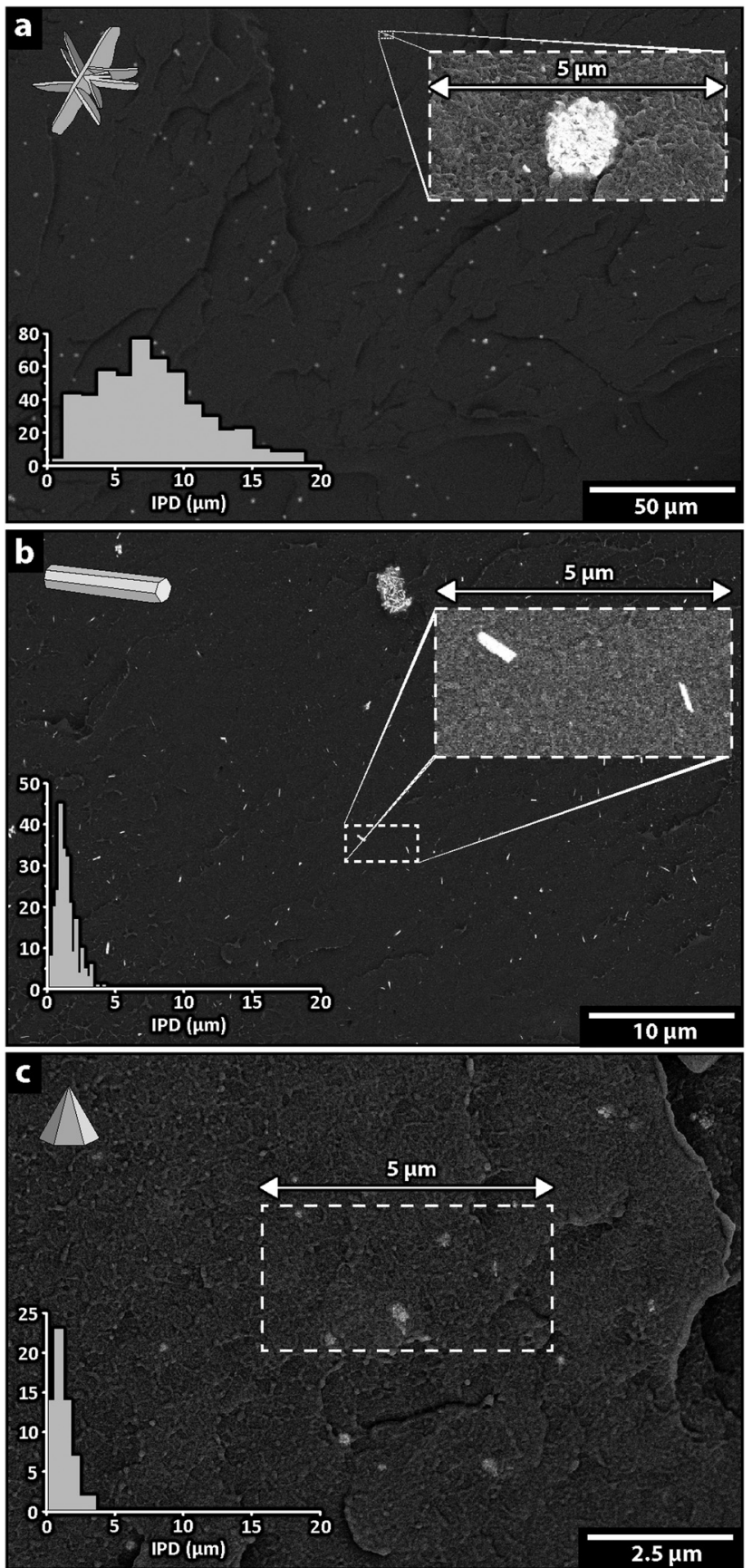

Fig. 8 Scanning electron micrographs of freeze-cracked cross-sections of LDPE composites containing (a) 3 wt \% unmodified ball-shaped particles, (b) 3 wt\% unmodified rod-shaped particles and (c) 3 wt\% unmodified nanoparticles. The histogram insets (bottom left) show the distribution of inter-particle distances obtained from several micrographs and the morphology of each particle is illustrated by the insets in the top left corner. A $5 \mu \mathrm{m}$ wide area is marked on all micrographs and a zoomed view is shown in the insets in (a) and (b).

wide dotted rectangle in Fig. 8c contains several agglomerates and a large number of nanoparticles. A more detailed image of these large agglomerates is shown in Fig. S7a (ESI†).

The average distance between the particles in a composite, commonly referred to as IPD (inter-particle distance), can be 
used to describe the degree of dispersion. ${ }^{15,16}$ The histogram in Fig. 8a shows that this distance ranged from 1 to $20 \mu \mathrm{m}$ with an average of $8 \mu \mathrm{m}$. The IPD value can also be used to assess the particle dispersion in agglomerated systems by considering distance between agglomerates. The histograms in Fig. 8b and $\mathrm{c}$ show IPD values ranging from sub- $\mu \mathrm{m}$ to a few microns, with an average of $c a .1 \mu \mathrm{m}$, indicating a very good dispersion of the rod-shaped particles but an extremely poor dispersion of the nanoparticles. Table 2 shows average IPD values for composites containing unmodified and coated $\mathrm{ZnO}$ particles, indicating that the dispersion was affected only when the C8 coating was applied to the nanoparticles. The IPD value for the nanoparticles was reduced 4 times (from 1.1-1.2 to $0.3 \mu \mathrm{m}$ ) with the hydrophobic coating. A typical dispersion of $\mathrm{C} 8$ modified nanoparticles is shown in Fig. S7b (ESI $\dagger$ ).

Fig. 9 shows the DC conductivity after $15 \mathrm{~h}$ in relation to the degree of dispersion of the different particles, using the average inter-particle distance IPD values. For the micro-sized particle composite systems, the IPD values, reflecting the degree of dispersion, did not significantly affect the conductivity of the composite and an increase in the IPD value from 1.4 to $7.8 \mu \mathrm{m}$ only resulted in a maximum 2 times increased conductivity (see 'Un' values in Fig. 9), regardless of the shape of the particles. The conductivities of both composites containing micro-sized particles were however significantly altered when a few nanometer thick $\mathrm{SiO}_{2}$ coating was applied, as indicated by the vertical increase in conductivity for the composites containing ball- or rod-shaped particles, marked in red and green, respectively. The coating resulted in an almost 10-fold increase in conductivity (see $\Delta$ in Fig. 9), due to the dominant and detrimental oxygen in the $\mathrm{SiO}_{2}$ coatings (see Sections 3.4 and 3.5).

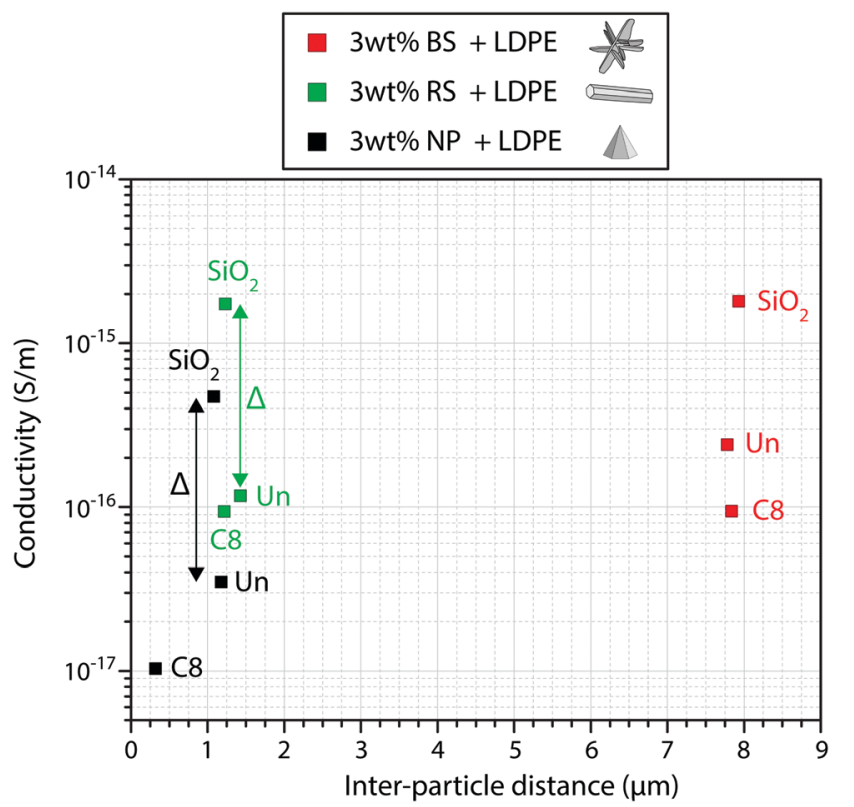

Fig. 9 Composite conductivity after $15 \mathrm{~h}$ of polarization as a function of the average inter-particle distance. Composites with unmodified particles are denoted 'Un'.
Table 2 Average inter-particle distance (IPD) for LDPE composites

\begin{tabular}{llll}
\hline $\begin{array}{l}\text { Surface } \\
\text { treatment }\end{array}$ & $\begin{array}{l}\text { IPD }(\mu \mathrm{m}) \text { BS } \\
\text { particles }\end{array}$ & $\begin{array}{l}\text { IPD }(\mu \mathrm{m}) \mathrm{RS} \\
\text { particles }\end{array}$ & $\begin{array}{l}\text { IPD }(\mu \mathrm{m}) \\
\text { nanoparticles }\end{array}$ \\
\hline Unmodified & 7.8 & 1.4 & 1.2 \\
Silica & 7.9 & 1.2 & 1.1 \\
Silsesquioxane $(\mathrm{C} 8)$ & 7.8 & 1.2 & 0.3
\end{tabular}

The agglomerated nanoparticle system containing the unmodified nanoparticles (with similar IPD as the rod-shaped particles: 1.2-1.4 $\mu \mathrm{m}$ ) displayed a lower conductivity than the larger unagglomerated rod-shaped particles $\left(3.5 \times 10^{-17}\right.$ and $1.2 \times$ $10^{-16} \mathrm{~S} \mathrm{~m}^{-1}$, respectively), showing that the nanoparticle surface is still active within the nanoparticle agglomerates. Introducing the $\mathrm{C} 8$ coating as a dispersion aid resulted in a relatively large drop in conductivity from $3.5 \times 10^{-17}$ to $1 \times$ $10^{-17} \mathrm{~S} \mathrm{~m}^{-1}$, compared to that of the composite system with larger particles. This reduction in conductivity was attributed to the inherent low current stabilizing particle interface LCSPI effect associated with the hydrocarbon terminations, accompanied by the significantly improved dispersion (reduced IPD values) of the high surface area nanoparticles, although the surface of the unmodified nanoparticles remained active as an interface in the aggregated state. The oxygen in the outer layers of the silica coating on the nanoparticles increased the composite conductivity by the same magnitude (see $\Delta$ in Fig. 9) as for the rod-shaped particles, despite the three times greater surface area of the $25 \mathrm{~nm}$ nanoparticles. This may be interpreted as being due to an encapsulation of multiple nanoparticles by the silica coating (possibly as coated aggregates) that consequently behave like large micro-sized particles or that there is an upper limit for the detrimental concentration effect of oxygen.

\section{Discussion}

Tanaka et $a l .{ }^{16}$ suggested a multicore model with a total interface thickness of 10-30 $\mathrm{nm}$ for particles $20-50 \mathrm{~nm}$ in size, while Smith et al. ${ }^{38}$ suggested an interface thickness of 10-20 nm considering the limited mobility of polymer chains in the vicinity of particles. It was also suggested that magnesium oxide $(\mathrm{MgO})$ particles in polyethylene have a maximum interface thickness of $40 \mathrm{~nm}$ according to calculations based on density functional theory. ${ }^{39}$ Takada et al. ${ }^{40}$ suggested that up to $100 \mathrm{~nm}$ trap wells are present for $100 \mathrm{~nm}$-large $\mathrm{MgO}$ particles at very high electric fields due to induced dipole moments. Intermodulation electrostatic force microscopy (ImEFM) has been used to map the surface potential on a nanometric scale at the particle interface, and this showed a halo around the particles consistent with a thickness of $c a .30 \mathrm{~nm}$ for modified $\mathrm{Al}_{2} \mathrm{O}_{3}$ nanoparticles in a LDPE composite. ${ }^{28}$ In essence, several works have pointed at a volumetric interphase extending radially $c a .20-50 \mathrm{~nm}$ from the inorganic surface of the particles.

In the present work it is suggested that the active particle interface moving the current towards lower values is much thinner than previously reported. The thickness of the low current stabilizing particle interface LCSPI is suggested to be 


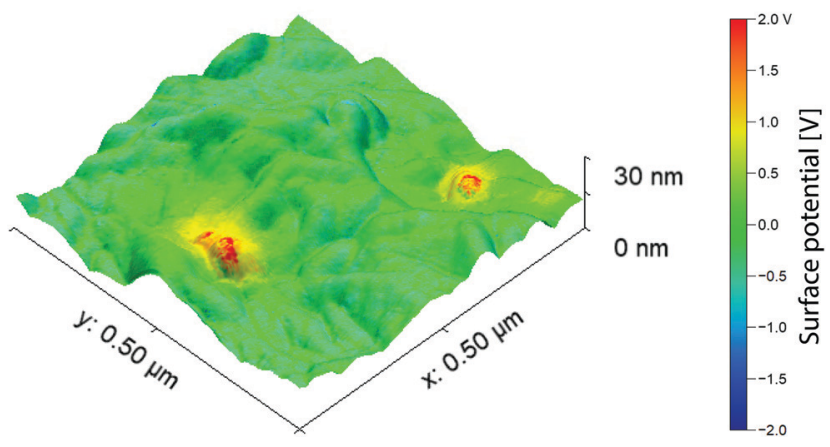

Fig. 10 Intermodulation electrostatic force microscopy of a spin coated $100 \mathrm{~nm}$ thick composite containing 3 wt\% C8-coated $\mathrm{ZnO}$ nanoparticles measured with a DC bias of $2 \mathrm{~V}$.

of the order of $1-2 \mathrm{~nm}$, which is sufficient to contain the C8 carbon functionality in the $\mathrm{SiO}_{X}$ particle coatings (Fig. 5 and 6). A critical zone up to $30 \mathrm{~nm}$ thick, surrounding the active interface may however work to accumulate charge at a slower rate than the charge transfers in the LCSPI, as previously suggested and visualized with electrostatic force microscopy.

Fig. 10 shows the topography on a nanoscale for a $500 \mathrm{~nm}$ wide area on a $100 \mathrm{~nm}$ thick polyethylene film containing C8-coated ZnO nanoparticles. The surface potential was measured by ImEFM, using a DC bias of $2 \mathrm{~V}$, corresponding to $20 \mathrm{kV} \mathrm{mm}^{-1}$. The absolute values were shifted to zero for the polyethylene matrix, the green color in the figure. The embedded $\mathrm{ZnO}$ nanoparticles are distinguished from the crystal lamellae structure by the red/yellow areas, associated with an almost $1 \mathrm{~V}$ positive charge extending tens of nanometers into the polymer matrix. The results of ImEFM measurements on all the $\mathrm{ZnO}$ nanocomposite materials are shown in Fig. S8 (ESI $\dagger$ ), for a bias of $-2,0$ and $2 \mathrm{~V}$. Although the samples showed a similar surface potential for the coated particles as in Fig. 10, the results could not however explain why the DC conductivity of composites containing silica-coated particles was one order of magnitude higher than that of the particles with aliphatic silsesquioxane coatings. This shows that the charge accumulation and transfer in nanoparticle coatings requires a more refined technique with a higher resolution to yield sufficient image visualization. No shift in surface potential related to the semicrystalline nature of the polymer matrix and differences in PE crystallinity between the samples was observed (Table S1, ESI $\dagger$ ). Mixtures of high-density and low-density polyethylene containing different lamellar thicknesses are known to affect the conductivity to a smaller extent than for metal-oxide nanocomposites. ${ }^{41}$ The crystalline PE structure in the vicinity of a ball-shaped particle was similar to that of the PE reference, with a measured average lamellae thickness of $8 \mathrm{~nm}$ for both materials (Fig. S9a and b, ESI $\dagger$ ).

\section{Conclusions}

Particle size, inter-particle distance, the presence of particle aggregates, the interface compatibility of polymer and inorganic phase, chemical residuals from the polymerization of the matrix, the presence of absorbed ambient humidity, and the properties of the particles are all factors that have been reported to affect the conductive nature of insulating dielectric nanocomposites..$^{1-3,5,15,42-44}$ Their relative contributions to the conductivity has however been mostly unknown, although it has been demonstrated that the greatest contribution to limiting electrical conduction in these materials has been provided by an inorganic nanoparticle phase organized into solitary particles and/or clusters (aggregates) of varying sizes. ${ }^{6}$ The extent to which the different factors, associated with the inorganic particle phase, affect the overall conductivity is in this work, extensively explored for composites of semicrystalline polyethylene. It is shown that the particle surface atoms in terms of the inorganic lattice terminations of the particles affect the conductivity significantly, and that the overall conductivity of the material can be controlled by selectively tailoring of the interfaces/ interphases in the particle filler material. This conclusion was possible to draw after eliminating the presence of particle agglomerates while comparing micro-sized $\mathrm{ZnO}$ particles with crystal faces either dominated with zinc or oxygen atoms. Composites containing particles with mostly oxygen surfaces showed significantly higher conductivity than composites with zinc terminations, 2.4 and $1.2 \times 10^{-16} \mathrm{~S} \mathrm{~m}^{-1}$, respectively. The effect of specific atomic terminations was confirmed by eliminating the difference in zinc and oxygen terminations by encapsulating the particles in $5 \mathrm{~nm}$ thick silicone-oxide-based coatings, resulting in identical electrical conductivities. However, silicone-oxide-based coatings consisting of silica resulted in 10 times more conductive composites $\left(2 \times 10^{-15} \mathrm{~S} \mathrm{~m}^{-1}\right)$ regardless of underlying $\mathrm{ZnO}$ terminations, while silsesquioxane coatings containing 8 carbon atom units long aliphatic chains suppressed the interface currents to $9.5 \times 10^{-17} \mathrm{~S} \mathrm{~m}^{-1}$, which is a marked lower conductivity than that of composites containing the 'as-synthesized' particles without any coatings (ca. $2 \times 10^{-16} \mathrm{~S} \mathrm{~m}^{-1}$ ). The results demonstrate that although oxygen has an overall negative impact when integrated in a coating, this can be compensated for by integration of $c a .1 \mathrm{~nm}$ organic carbon functional units, herein referred to as a low current stabilizing particle interface (LCSPI), suppressing the conductivity of nanodielectric composites. The phenomenon described was applicable to composites containing $25 \mathrm{~nm}$ sized ZnO nanoparticles, which in combination with the aliphatic carbon terminations displayed the lowest conductivity of all the explored systems $\left(1 \times 10^{-17} \mathrm{~S} \mathrm{~m}^{-1}\right)$.

The development of future low conductive nanodielectrics, for the more extensive insulation of HVDC cables and other applications, will ultimately rely on establishing robust protocols for the preparation of controlled compositions of the nanometric particle coating interface. The challenge will here be to design these coatings so they simultaneously allow for controlling the dispersion of the nanoparticles in largescale nanodielectrics manufacture. Overall, the results suggest however that the interphase control of the inorganic particles dictate the dielectric properties of the nanocomposite before the interphase of any types of crystals present in the polyethylene. 


\section{Conflicts of interest}

There are no conflicts of interest to declare.

\section{Acknowledgements}

This work was funded through SweGRIDS, by the Swedish Energy Agency and ABB.

\section{References}

1 A. M. Pourrahimi, T. A. Hoang, D. M. Liu, L. K. H. Pallon, S. Gubanski, R. T. Olsson, U. W. Gedde and M. S. Hedenqvist, Highly Efficient Interfaces in Nanocomposites Based on Polyethylene and ZnO Nano/Hierarchical Particles: A Novel Approach toward Ultralow Electrical Conductivity Insulations, Adv. Mater., 2016, 28, 8651-8657.

2 L. K. H. Pallon, A. T. Hoang, A. M. Pourrahimi, M. S. Hedenqvist, F. Nilsson, S. Gubanski, U. W. Gedde and R. T. Otsson, The impact of MgO nanoparticle interface in ultra-insulating polyethylene nanocomposites for high voltage DC cables, J. Mater. Chem. A, 2016, 4, 8590-8601.

3 D. Liu, A. T. Hoang, A. M. Pourrahimi, L. K. H. Pallon, F. Nilsson, S. M. Gubanski, T. Olsson, M. S. Hedenqvist and U. W. Gedde, Influence of Nanoparticle Surface Coating on Electrical Conductivity of LDPE/Al2O3 Nanocomposites for HVDC Cable Insulations, IEEE Trans. Dielectr. Electr. Insul., 2017, 24, 1396-1404.

4 P. Bergelin, M. Jeroense, T. Quist and H. Rapp, $640 \mathrm{kV}$ Extruded HVDC Cable System, Technical Paper, NKT, 2017.

5 T. Hjertberg, V. Englund, P. Hagstrand, W. Loyens, U. Nilsson and A. Smedberg, Materials for HVDC cables, Jicable HVDC'13, France, 2013, pp. 11-15.

6 A. M. Pourrahimi, R. T. Olsson and M. S. Hedenqvist, The Role of Interfaces in Polyethylene/Metal-Oxide Nanocomposites for Ultrahigh-Voltage Insulating Materials, Adv. Mater., 2018, 30, 1703624.

7 J. C. Fothergill, Ageing, Space Charge and Nanodielectrics: Ten Things We Don't Know About Dielectrics, 2007 IEEE International Conference on Solid Dielectrics, 2007, vol. 1 and 2, pp. 1-10, DOI: 10.1109/Icsd.2007.4290739.

8 G. Teyssedre and C. Laurent, Charge transport modeling in insulating polymers: From molecular to macroscopic scale, IEEE Trans. Dielectr. Electr. Insul., 2005, 12, 857-875.

9 T. J. Lewis, Interfaces: nanometric dielectrics, J. Phys. D: Appl. Phys., 2005, 38, 202-212.

10 Y. J. Cheng, L. Y. Bai, G. Yu and X. H. Zhang, Effect of Particles Size on Dielectric Properties of Nano-ZnO/LDPE Composites, Materials, 2019, 12, 5.

11 K. Ishimoto, E. Kanegae, Y. Ohki, T. Tanaka, Y. Sekiguchi, Y. Murata and C. C. Reddy, Superiority of Dielectric Properties of LDPE/MgO Nanocomposites over Microcomposites, IEEE Trans. Dielectr. Electr. Insul., 2009, 16, 1735-1742.

12 J. K. Nelson, J. C. Fothergill, L. A. Dissado and W. Peasgood, Towards an understanding of nanometric dielectrics,
Annual Report Conference on Electrical Insulation and Dielectric Phenomena, 2002, pp. 295-298.

13 C. Calebrese, L. Hui, L. S. Schadler and J. K. Nelson, A Review on the Importance of Nanocomposite Processing to Enhance Electrical Insulation, IEEE Trans. Dielectr. Electr. Insul., 2011, 18, 938-945.

14 S. T. Li, G. L. Yin, G. Chen, J. Y. Li, S. N. Bai, L. S. Zhong, Y. X. Zhang and Q. Q. Lei, Short-term Breakdown and Long-term Failure in Nanodielectrics: A Review, IEEE Trans. Dielectr. Electr. Insul., 2010, 17, 1523-1535.

15 D. Liu, A. M. Pourrahimi, R. T. Olsson, M. S. Hedenqvist and U. W. Gedde, Influence of nanoparticle surface treatment on particle dispersion and interfacial adhesion in low-density polyethylene/aluminium oxide nanocomposites, Eur. Polym. J., 2015, 66, 67-77.

16 T. Tanaka, M. Kozako, N. Fuse and Y. Ohki, Proposal of a multi-core model for polymer nanocomposite dielectrics, IEEE Trans. Dielectr. Electr. Insul., 2005, 12, 669-681.

17 J. L. Castro-Mayorga, M. J. Fabra, A. M. Pourrahimi, R. T. Olsson and J. M. Lagaron, The impact of zinc oxide particle morphology as an antimicrobial and when incorporated in poly(3-hydroxybutyrate-co-3-hydroxyvalerate) films for food packaging and food contact surfaces applications, Food Bioprod. Process., 2017, 101, 32-44.

18 A. M. Pourrahimi, D. Liu, L. K. H. Pallon, R. L. Andersson, A. M. Abad, J. M. Lagaron, M. S. Hedenqvist, V. Ström, U. W. Gedde and R. T. Olsson, Water-based synthesis and cleaning methods for high purity $\mathrm{ZnO}$ nanoparticles comparing acetate, chloride, sulphate and nitrate zinc salt precursors, RSC Adv., 2014, 4, 35568-35577.

19 A. M. Pourrahimi, D. Liu, V. Strom, M. S. Hedenqvist, R. T. Olsson and U. W. Gedde, Heat treatment of $\mathrm{ZnO}$ nanoparticles: new methods to achieve high-purity nanoparticles for high-voltage applications, J. Mater. Chem. A, 2015, 3, 17190-17200.

20 A. M. Pourrahimi, D. M. Liu, R. L. Andersson, V. Strom, U. W. Gedde and R. T. Olsson, Aqueous Synthesis of (210) Oxygen-Terminated Defect-Free Hierarchical ZnO Particles and Their Heat Treatment for Enhanced Reactivity, Langmuir, 2016, 32, 11002-11013.

21 M. E. Karlsson, Y. C. Mamie, A. Calamida, J. M. Gardner, V. Strom, A. M. Pourrahimi and R. T. Olsson, Synthesis of Zinc Oxide Nanorods via the Formation of Sea Urchin Structures and Their Photoluminescence after Heat Treatment, Langmuir, 2018, 34, 5079-5087.

22 N. M. Macleod, M. Callavik, M. Boden, M. Dhesi, R. Huuva, N. Kuljaca, F. Schettler and P. Brinckerhoff, A Technological Roadmap for the Development of the European Supergrid, Cigré Int. Symp. - Across Borders - HVDC Syst. Mark. Integr., Lund, Sweden, 2015.

23 A. P. Gray, Polymer crystallinity determinations by DSC, Thermochim. Acta, 1970, 1, 563-579.

24 B. Wunderlich and H. Bauer, Heat Capacities of Linear High Polymers, Adv. Polym. Sci., 1970, 7, 151.

25 B. Wunderlich, Heat of Fusion of Polyethylene, J. Polym. Sci., 1967, 5(Pt A2), 987-988. 
26 H. Ghorbani, T. Christen, M. Carlen, E. Logakis, L. Herrmann, H. Hillborg, L. Petersson and J. Viertel, Longterm Conductivity Decrease of Polyethylene and Polypropylene Insulation Materials, IEEE Trans. Dielectr. Electr. Insul., 2017, 24, 1485-1493.

27 R. Borgani, D. Forchheimer, J. Bergqvist, P. A. Thoren, O. Inganas and D. B. Haviland, Intermodulation electrostatic force microscopy for imaging surface photo-voltage, Appl. Phys. Lett., 2014, 105, 143113.

28 R. Borgani, L. K. H. Pallon, M. S. Hedenqvist, U. W. Gedde and D. B. Haviland, Local Charge Injection and Extraction on Surface-Modified $\mathrm{Al} 2 \mathrm{O} 3$ Nanoparticles in LDPE, Nano Lett., 2016, 16, 5934-5937.

29 S. Cho, J. W. Jang, J. S. Lee and K. H. Lee, Exposed Crystal Face Controlled Synthesis of 3D ZnO Superstructures, Langmuir, 2010, 26, 14255-14262.

30 B. X. Li and Y. F. Wang, Facile Synthesis and Enhanced Photocatalytic Performance of Flower-like ZnO Hierarchical Microstructures, J. Phys. Chem. C, 2010, 114, 890-896.

31 G. Ambrosetti, N. Johner, C. Grimaldi, T. Maeder, P. Ryser and A. Danani, Electron tunneling in conductor-insulator composites with spherical fillers, J. Appl. Phys., 2009, 106, 016103.

32 G. C. Li, G. Chen and S. T. Li, Charge transport model in nanodielectric composites based on quantum tunneling mechanism and dual-level traps, Appl. Phys. Lett., 2016, 109, 062901.

33 J. R. Winkler and H. B. Gray, Long-Range Electron Tunneling, J. Am. Chem. Soc., 2014, 136, 2930-2939.

34 X. Wang, J. K. Nelson, L. S. Schadler and H. Hillborg, Mechanisms Leading to Nonlinear Electrical Response of a Nano p-SiC/Silicone Rubber Composite, IEEE Trans. Dielectr. Electr. Insul., 2010, 17, 1687-1696.

35 D. Liu, A. M. Pourrahimi, L. K. H. Pallon, R. L. Andersson, M. S. Hedenqvist, U. W. Gedde and R. T. Olsson, Morphology and properties of silica-based coatings with different functionalities for $\mathrm{Fe}_{3} \mathrm{O}_{4}, \mathrm{ZnO}$ and $\mathrm{Al}_{2} \mathrm{O}_{3}$ nanoparticles, $R S C$ Adv., 2015, 5, 48094-48103.
36 R. T. Olsson, M. S. Hedenqvist, V. Strom, J. Deng, S. J. Savage and U. W. Gedde, Core-Shell Structured FerriteSilsesquioxane-Epoxy Nanocomposites: Composite Homogeneity and Mechanical and Magnetic Properties, Polym. Eng. Sci., 2011, 51, 862-874.

37 J. Coates, in Encyclopedia of Analytical Chemistry, ed. R. A. Meyers, John Wiley \& Sons Ltd, Chichester, 2000, pp. 1081510837.

38 R. C. Smith, C. Liang, M. Landry, J. K. Nelson and L. S. Schadler, The mechanisms leading to the useful electrical properties of polymer nanodielectrics, IEEE Trans. Dielectr. Electr. Insul., 2008, 15, 187-196.

39 E. Kubyshkina, M. Unge and B. L. G. Jonsson, Communication: Band bending at the interface in polyethylene-MgO nanocomposite dielectric, J. Chem. Phys., 2017, 146, 051101.

40 T. Takada, Y. Hayase, Y. Tanaka and T. Okamoto, Space charge trapping in electrical potential well caused by permanent and induced dipoles for LDPE/MgO nanocomposite, IEEE Trans. Dielectr. Electr. Insul., 2008, 15, 152-160.

41 M. G. Andersson, J. Hynynen, M. R. Andersson, V. Englund, P. O. Hagstrand, T. Gkourmpis and C. Muller, Highly Insulating Polyethylene Blends for High-Voltage DirectCurrent Power Cables, ACS Macro Lett., 2017, 6, 78-82.

42 M. Roy, J. K. Nelson, R. K. MacCrone and L. S. Schadler, Candidate mechanisms controlling the electrical characteristics of silica/XLPE nanodielectrics, J. Mater. Sci., 2007, 42, 3789-3799.

43 M. Praeger, I. L. Hosier, A. F. Holt, A. S. Vaughan and S. G. Swingler, On the Effect of Functionalizer Chain Length and Water Content in Polyethylene/Silica Nanocomposites: Part II-Charge Transport, IEEE Trans. Dielectr. Electr. Insul., 2017, 24, 2410-2420.

44 F. Nilsson, M. Karlsson, L. Pallon, M. Giacinti, R. T. Olsson, D. Venturi, U. W. Gedde and M. S. Hedenqvist, Influence of water uptake on the electrical DC-conductivity of insulating LDPE/MgO nanocomposites, Compos. Sci. Technol., 2017, 152, 11-19. 